\title{
Sensitive and Accurate Dispersion Map Extraction of HNLFs by Frequency Tuning of a Degenerate FWM
}

\author{
Fatemeh Alishahi, Armand Vedadi, Mohammad A. Shoaie, Camille-Sophie Brès, and Khashayar Mehrany
}

\begin{abstract}
A sensitive and accurate method for dispersion map extraction along an arbitrarily profiled highly nonlinear fiber (HNLF) is proposed. High sensitivity is achieved by positioning the wavelength of a signal at the band edge of the modulation instability (MI) spectrum generated by an intense degenerate four wave mixing (FWM) pump. In doing so, the to-be-extracted dispersion fluctuations leave a more drastic effect on the FWM-generated power since they either inflate or deflate the MI spectrum. The accuracy of the method is increased by monitoring the distribution of power along the fiber. Once the power distribution is measured along the fiber, dispersion map of the HNLF is extracted using an appropriate inverse algorithm, which reconstructs the dispersion with a highlevel ofaccuracy.
\end{abstract}

Index Terms-Brillouin optical time domain analysis, dispersion map, four wave mixing, highly nonlinear fiber, parametric amplification, zero-dispersion wavelength.

\section{INTRODUCTION}

$\mathbf{H}$ IGHLY Nonlinear Fibers, (HNLFs), can be fabricated with dispersion properties which enhance nonlinear interactions based on four-wave mixing (FWM) within the telecommunication band [1]-[4]. The nonlinear effects are generated with relatively low input powers thanks to the large nonlinear coefficient of the HNLF due to its small mode effective area and high amount of dopants [5]. Despite the remarkable level of maturity in the fabrication process of HNLFs [3], [6], [7], the dispersion characteristics along the fiber are significantly altered by the inevitable microscopic variations of the HNLF cross section [5]. The random fluctuations of the dispersion characteristics, even when they are as short as few meters, strongly affect the phase matching condition and thereby degrade the overall performance of FWM processes [7]-[10]. Fortunately, compensation schemes are available to counterbalance the phase matching fluctuations caused by dispersion fluctuations along the fiber [11]-[13]. An efficient compensation scheme for HNLF, however, requires having reliable dispersion map along the fiber [6], [7], [12]-[14]. One tenable methodology for extraction of the dispersion map is to initiate a

Manuscript received March 19, 2016; revised July 8, 2016; accepted August 10, 2016. Date of publication August 16, 2016; date of current version September 2, 2016. This work was supported by Marie Curie CIG under Grant 303700.

F. Alishahi and K. Mehrany are with the Department of Electrical Engineering, Sharif University of Technology, Tehran Iran (e-mail: alishahi.fateme@ gmail.com; mehrany@sharif.edu).

A. Vedadi, M. A. Shoaie, and C.-S. Brès are with the Photonics System Laboratory, Ecole Polytechnique Fédérale de Lausanne, Lausanne CH-1015, Switzerland (e-mail: avedadi@gmail.com; mashoaie@gmail.com; camille. bres@epfl.ch).

Color versions of one or more of the figures in this paper are available online at http://ieeexplore.ieee.org.

Digital Object Identifier 10.1109/JLT.2016.2601064 type of localized nonlinear interaction and the sought-after dispersion map can be extracted nondestructively by monitoring the localized interaction [6], [14]. Generally, this methodology becomes convoluted when the efficiency of the nonlinear interaction is predominantly determined by the nonlinear coefficient of the fiber rather than the to-be-extracted dispersion. This is what handicaps dispersion map extraction of HNLFs. Not surprisingly, the only present scheme for extraction of dispersion map is the elegant and elaborate work of Myslivets et al. [6], [7], [15]. Although, this interesting method provides sub-meter scale resolution, it is inevitably complicated both from the experimental [5], [7] and dispersion reconstruction algorithm [15] viewpoints.

Basically, the extracted dispersion map using localized nonlinear interaction would have two resolution features; one is the physical spatial resolution or simply the resolution, which defines the minimum distance of resolvable points along the fiber axis. Second is the vertical resolution which relates to minimum resolvable difference between the values of dispersions at two consecutive spatial points. Although, any spatial frequency concept such as dispersion would inevitably feature a theoretical spatial resolution as well, it may be disregard for being negligible compared to the meter-scale physical spatial resolution. Though, the physical spatial resolution of extracted dispersion is simply the spatial resolution of the measurements, the vertical resolution of dispersion map is not directly accessible. It can only be assessed in any dispersion map extraction scheme via the vertical resolution of the powers of the localized interactions. However, as the power of waves at a specific point along the fiber depends on the average of dispersion over the propagation length [16], [17], the vertical resolution of the dispersion is poorly reflected in the vertical resolution of the measured power, in general. Therefore, it is crucial to reach to a scheme that enlarges the effect of dispersion vertical resolution and reflects it in the vertical resolution of nonlinear localized interaction or in other words enhances the sensitivity.

In this paper, for the first time to the best of our knowledge a less complex yet highly sensitive approach is proposed for extraction of dispersion map in HNLFs. The success of the proposed method is indebted to the following facts. First, the efficiency of the nonlinear interaction in which the dispersion fluctuations of the fiber are reflected is measured by monitoring the distribution of the generated FWM power along the fiber instead of optical power measurement at the fiber output [6]. However, distributed power measurements would not necessarily render enough sensitivity for HNLF dispersion extraction. Therefore as the second fact, the wavelengths of the interacting waves are carefully chosen to ensure that the sensitivity 
of the scheme is enhanced. As the result of high sensitivity, the improved accuracy of the method is assessed using the simulatethen-retrieve procedure [15].

The remainder of this paper is organized as follows. Initially in Section II, theory of FWM interaction under dispersion fluctuations is introduced. Then, the proposed method for the derivation of dispersion map is explained in Section III. In Section IV, the method introduced in Section III is numerically studied and its enhanced sensitivity and as the result, high accuracy for dispersion reconstruction are demonstrated using numerical simulations. After that, the obtained results from the experimental setup introduced in Section III, are presented and validated in Section V. Finally, the conclusions are made in Section VI.

\section{THEORETICAL FOUNDATION}

One conceivable method to extract the dispersion map of an HNLF in a non-destructive fashion is to call upon nonlinear interaction whose efficiency depends on dispersion. In a degenerate FWM process, the dispersion fluctuation at signal and idler frequencies counterbalance each other in the linear phase mismatch term of the process while the contribution of the dispersion fluctuation at the pump frequency remains intact [16], [17]. This fact taken at its face value suggests that extraction of the dispersion fluctuation at the pump frequency is straightforward. Regrettably, however, the efficiency of the nonlinear process is not solely determined by the linear phase mismatch term. Therefore, extraction of dispersion fluctuations at the pump frequency is not as straightforward as one might expect. This fact is better understood by studying the following equations governing the power distribution of pump, $P_{p}(z)$, and two sidebands which are hereafter referred to as the signal, $P_{s}(z)$, and the idler, $P_{i}(z)$ :

$$
\begin{aligned}
& d P_{p} / d z=2 \gamma P_{i} P_{s} \sin (\theta) \\
& d P_{s} / d z=-\gamma P_{p} P_{i} \sin (\theta) \\
& d P_{i} / d z=-\gamma P_{p} P_{s} \sin (\theta)
\end{aligned}
$$

In these expressions, $\gamma$ is the nonlinear coefficient of the fiber and is assumed to be uniform along the fiber. It should be noted that the HNLF loss has not been taken into account [6], [15] as a result of typical short length. The pump, signal, and idler are frequency matched but are not necessarily phase matched. Their relative phase mismatch $\theta(z)$ is as follows and determines the efficiency of their nonlinear interaction [16], [17]:

$$
\theta(z)=\theta_{l}(z)+\theta_{n}(z)+\theta(0)
$$

where

$$
\begin{aligned}
\theta_{n}(z)= & \gamma \int_{0}^{z} 2 P_{p}-P_{s}-P_{i} \\
& +P_{p}\left(\sqrt{\frac{P_{i}}{P_{s}}}+\sqrt{\frac{P_{s}}{P_{i}}}-\frac{4 \sqrt{P_{i} P_{s}}}{P_{p}}\right) \cos (\theta) d z^{\prime}
\end{aligned}
$$

and

$$
\theta_{l}(z)=\int_{0}^{z} \Delta \beta\left(z^{\prime}\right) d z^{\prime}
$$

These expressions show that the complexity of extracting dispersion fluctuations at the pump wavelength is due to the following two factors. First, the sought-after dispersion map at the pump wavelength $\lambda_{p}$ along the fiber, $D\left(z, \lambda_{p}\right)$, contributes in the linear term of the overall relative phase mismatch, $\theta_{l}(z)$, via the integral of the linear propagation constant mismatch $\Delta \beta(z)$ :

$$
\Delta \beta(z) \approx-\frac{2 \pi c}{\lambda_{P}^{2}} D\left(z, \lambda_{P}\right) \Delta \lambda^{2}
$$

where $c$, and $\Delta \lambda$ are the speed of light at free space, and the wavelength detuning of pump and signal/idler, respectively. Therefore, single measurement of the optical power at the fiber endpoint does not provide the desired dispersion map $D\left(z, \lambda_{p}\right)$, but its integral along the fiber. Second, $\gamma P_{P}$ is usually a noticeable term in HNLFs such that nonlinear power interaction represents a non-negligible contribution to the overall relative phase mismatch.

The abovementioned issues can be addressed by taking the following measures. First, the number of optical power measurements can be increased to provide further information, which is needed to find the integrand, i.e., the linear propagation constant mismatch $\Delta \beta$, from the integral, i.e., the relative phase mismatch $\theta(z)$. Second, the contribution of the linear relative phase mismatch term, $\theta_{l}(z)$, in the nonlinear power interaction efficiency should be enhanced considerably. In what follows we introduce an experimental plan that resolves both mentioned issues.

\section{The Proposed Method For Extraction of Dispersion}

The idea of localized nonlinear interaction [6], [7], [18]-[22] can be implemented to extract the dispersion map of HNLFs. The basic principle is to initiate a localized nonlinear interaction whose efficiency depends on the dispersion characteristics of the spot at which the interaction is taking place. The efficiency of the nonlinear interaction is implicitly measured at either ends of the fiber, and the sought-after dispersion map is extracted by sweeping the nonlinear interaction spot along the fiber. The localized nonlinear interaction can be initiated by either two co-directional waves with different velocities [19], or two contra-directional waves colliding each other [6], [20][22]. The latter strategy is adopted here since it can reach the meter-scale resolution [6], [20] while the resolution of the earlier strategy is limited to the kilometer scales [19]. There are number of elegant works [20]-[22] which have used nonlinear localized interaction between contra-directional waves, and successfully extracted the dispersion map of SMFs or DSFs. However, these methods are not useful for nearly dispersion-less HNLFs since localized nonlinear interaction in such fibers is not sensitive enough to dispersion characteristics [6].

Our proposed method is based on Brillouin Optical TimeDomain Analysis (BOTDA) [23], which counts among optical time domain reflectometry techniques [24]. It has been already implemented for the realization of localized nonlinear interaction between contra-directional waves [25]-[27]. The basic idea is that a pulsed wave acts as a pump for Brillouin interaction with 


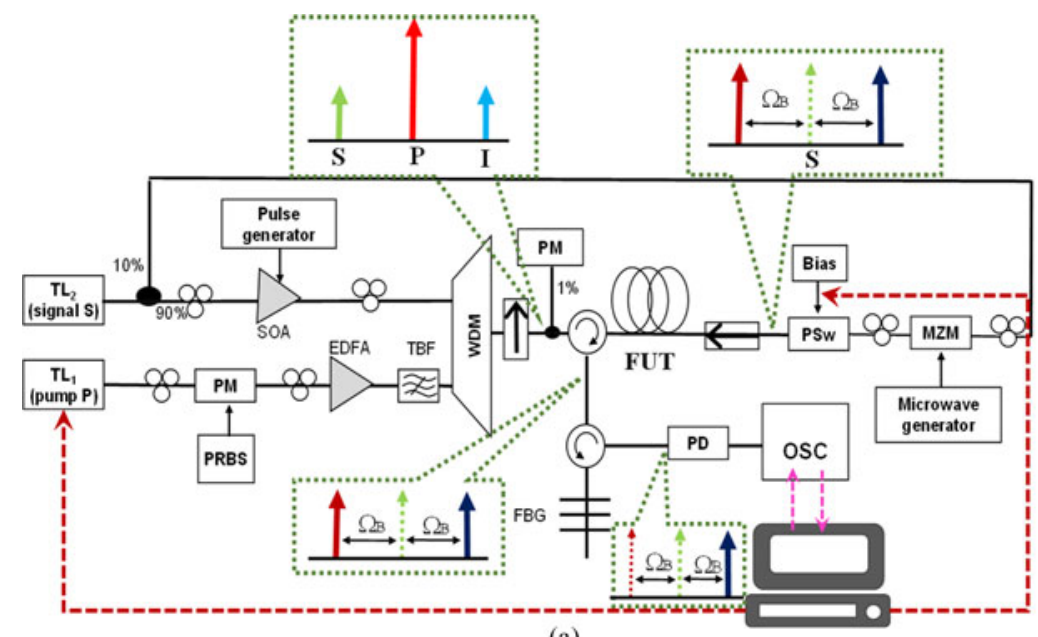

(a)



(b)

Fig. 1. (a) Experimental setup of the proposed scheme. PD: Photo-detector. The insets show the spectral components at each point. (b) Polarization locking scheme for parametric interaction.

a counter-propagating continuous-wave (CW) probe [25]-[27]. Using BOTDA idea, proposed experimental plan is developed which can effectively extract the dispersion map along HNLFs.

The proposed experimental setup is schematically shown in Fig. 1(a). An intense CW pump is generated by a tunable laser (TL1) and phase modulated by a $10-\mathrm{GHz}$ pseudo-random bit sequence. An erbium-doped fiber amplifier (EDFA) followed by a tunable filter, then boosts the pump to the fiber under test. The pulsed Brillion pump and two $\mathrm{CW}$ probes share the same laser source (TL2). The pulsed Brillouin pump is produced by periodically gating a semiconductor optical amplifier (SOA) for almost $80 \mathrm{~ns}$ at a period of $6 \mu \mathrm{s}$. This pulse duration corresponds to almost 8 meter spatial resolution. Using a wavelength-division multiplexer (WDM) the Brillouin pump is combined with the $\mathrm{CW}$ pump. They both pass through a polarizer and are injected into the same end of the fiber under test (FUT). The CW pump wavelength is adjusted slightly above zero- dispersion wavelength (ZDW) and thus modulation instability (MI) bumps appear at the fiber end [17]. Moreover, since the Brillouin pump is considerably weaker than the CW pump and its wavelength lies within the MI spectrum of the CW pump, both waves interact [15], [16] and generate an idler wave through Eqs. (1) and (2). The CW pump, the Brillouin and the generated waves are hereafter referred to as the FWM pump, the signal and the idler, respectively. They travel along the FUT, and are monitored in an optical spectrum analyzer (OSA).

The other portion of TL2 is carrier-suppressed-intensitymodulated by a CW tone with frequency of $9.8 \mathrm{GHz}$. This frequency is approximately matched to the average Brillouin frequency shift of the fiber, $\mathrm{W}_{\mathrm{B}}$. Hence, the two generated $\mathrm{CW}$ sidebands referred to as probe waves have the frequency detuning $\mathrm{W}_{\mathrm{B}}$ with the signal pulse. As pointed out in our previous work [26], the double sideband probe configuration provides far more robustness and accuracy. A polarization switch (PSw) switches the probe polarization between two orthogonal states by having its bias changed from $+5 \mathrm{~V}$ to $-5 \mathrm{~V}$ and vice versa. After passing through the PSw, the probe waves are routed to the opposite end of the fiber. Finally, one of the probe waves is selected by a fiber Bragg grating (FBG) and is routed to the oscilloscope (OSC).

Also, a programmable procedure has been run to ensure the alignment of the parametric pump and signal polarizations [26]. The basic of this procedure is graphically demonstrated in Fig. 1(b). In four runs of the experiment, the probe powers are captured in OSC and sent to a processor. In the first two runs, the power traces of either of the probe sidebands for two states of PSw are obtained while the FWM pump is on. Measured traces of OSC relating to the probe power at location $z=0$ of fiber can be written as [25], [28]:

$$
\begin{aligned}
& \tilde{P}_{H}^{O N}(0, t)=P_{0} \times \exp \left(\mp \int_{c(t+T) / 2}^{c(t-T) / 2} g_{B}^{H}(z) P_{s}(z) d z\right)(4-\mathrm{a}) \\
& \tilde{P}_{V}^{O N}(0, t)=P_{0} \times \exp \left(\mp \int_{c(t+T) / 2}^{c(t-T) / 2} g_{B}^{V}(z) P_{s}(z) d z\right)(4-\mathrm{b})
\end{aligned}
$$

where $g_{B}^{H}(z)$ or $g_{B}^{v}(z)$ denotes the Brillouin coefficients [29] for either of polarization states of the probe, $P_{s}(z)$ is the signal power along the fiber, $P_{0}$ represents the input power of the $\mathrm{CW}$ probes and $T$ stands for the signal pulse width. The plus and minus signs account for the Brillouin gain and loss, respectively, as one of the probes experiences Brillouin gain and the other Brillouin loss. In the last two runs of the experiment, the time variations of the probe sidebands at OSC are measured when the FWM pump is turned off and the PSw is in either of its polarization states. These powers recorded at point $z=0$ via the OSC, are:

$$
\begin{aligned}
& \tilde{P}_{H}^{O F F}(0, t)=P_{0} \times \exp \left(\mp \int_{c(t+T) / 2}^{c(t-T) / 2} g_{B}^{H}(z) P_{s}(0) d z\right)(5-\mathrm{a}) \\
& \tilde{P}_{V}^{O F F}(0, t)=P_{0} \times \exp \left(\mp \int_{c(t+T) / 2}^{c(t-T) / 2} g_{B}^{V}(z) P_{s}(0) d z\right)(5-\mathrm{b})
\end{aligned}
$$

In the above equations, $P_{s}(0)$ is the input peak power of the signal. It can be easily verified that the exponentials in Eqs. (4) and (5) can be approximated by their first order Taylor series 
expansion [25]. That is:

$$
\tilde{P}_{i}^{j}(0, t) \approx P_{0} \times\left(1 \mp \int_{c(t+T) / 2}^{c(t-T) / 2} g_{B}^{i}(z) P_{s}(0) d z\right)
$$

in which, $i=H, V$ and $j=O N, O F F$. Therefore, for zero average powers, $P_{i}^{j}(0, t) \approx \tilde{P}_{i}^{j}(0, t)-P_{0}$, the following equality holds:

$$
\frac{P_{H}^{O N}(0, t)+P_{V}^{O N}(0, t)}{P_{H}^{O F F}(0, t)+P_{V}^{O F F}(0, t)}=\frac{\left.\int_{c(t+T) / 2}^{c(t-T) / 2} g_{B}^{(} z\right) P_{s}(z) d z}{\left.\int_{c(t+T) / 2}^{c(t-T) / 2} g_{B}^{(} z\right) d z}
$$

in which $g_{B}(z)=g_{B}^{H}(z)+g_{B}^{v}(z)$ is the scalar Brillouin gain coefficient [29]. For short signal pulses, i.e. for small $c T$ values, Eq. (7) yields the normalized signal power, $\eta(z)=$ $P_{s}(z) / P(0)$, as:

$$
\frac{P_{H}^{O N}(0, t)+P_{V}^{O N}(0, t)}{P_{H}^{O F F}(0, t)+P_{V}^{O F F}(0, t)} \approx \eta(z=c t / 2)
$$

Now, $\eta(z)$ obtained by Eq. (8) can be attributed to the dispersion coefficient via Eqs. (1) and (2), provided that polarizations of the signal, FWM pump and the generated idler inside FUT are aligned. If signal is not completely aligned to the pump at fiber input, its polarization wanders randomly inside the fiber due to the pump pulling effect [30]. As a result, the parametric gain $\eta(z)$ in each run of experiment is not the same. Therefore, a sanity check for the polarization alignment is to inspect whether following equality is satisfied [26]:

$$
\begin{aligned}
& \frac{P_{H}^{O N}(0, t)+P_{V}^{O N}(0, t)}{P_{H}^{O F F}(0, t)+P_{V}^{O F F}(0, t)}=0.5 \\
& \quad \times\left[\frac{P_{H}^{O N}(0, t)}{P_{H}^{O F F}(0, t)}+\frac{P_{V}^{O N}(0, t)}{P_{V}^{O F F}(0, t)}\right]
\end{aligned}
$$

Therefore as depicted in Fig. 1(b), the four mentioned measured traces are recorded and processed to see whether Eq. (9) is satisfied. If not, the polarization controllers in the path of the signal and pump are retuned until Eq. (9) is satisfied.

\section{Numerical Study of the Proposed Method}

As already pointed out in Section II, the efficiency of the nonlinear interaction between the FWM pump and the signal depends not only on the dispersion map but also on nonlinear coefficient of the fiber. Therefore, it is tried to seek for a spectral regime in which the vertical resolution in dispersion produces a large vertical resolution in the generated FWM power or in other words, an enhanced sensitivity is achieved. In this order, numerical simulation of Eqs. (1) and (2) under different circumstances is carried out. A virtual fiber of length $270 \mathrm{~m}$ and nonlinear coefficient $\gamma=14.5 \mathrm{~W}^{-1} \mathrm{~km}^{-1}$ is considered. The dispersion coefficient of this fiber at the free space wavelength $\lambda=1558 \mathrm{~nm}$ is supposed to be $D(z, \lambda=1558 \mathrm{~nm})=D_{0}(\lambda)+\delta D(z)$, where $D_{0}(\lambda)=0.077 \mathrm{ps} /(\mathrm{nm} \cdot \mathrm{km})$ is the average dispersion coefficient along the fiber, and $\delta D(z)$ is an arbitrary dispersion fluctuation. $\delta D(z)$ as plotted by solid line in Fig. 2 is randomly generated in a very similar method as in [15] by summing up five sinusoids. To extract the dispersion fluctuations at $1558 \mathrm{~nm}$,

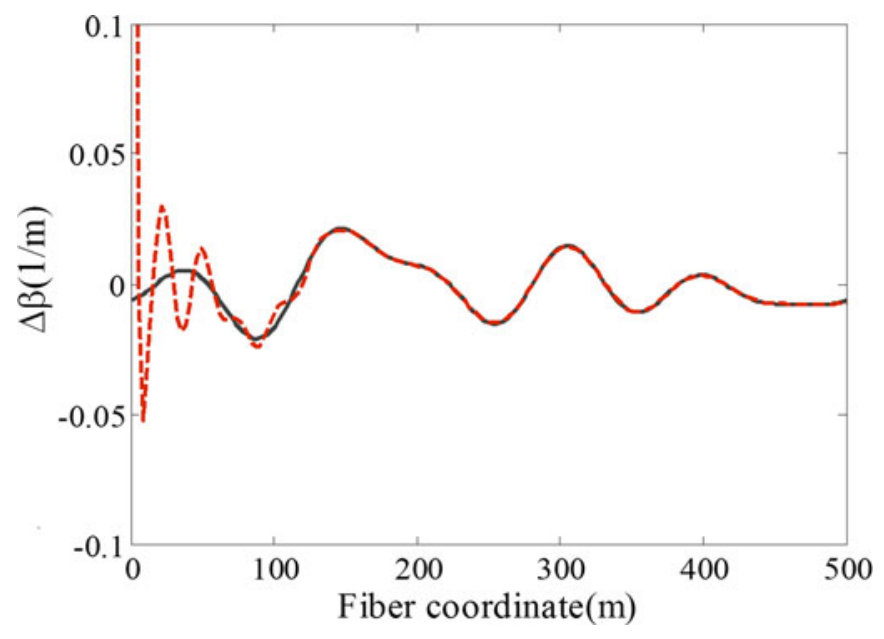

Fig. 2. Solid line: Randomly generated propagation constant mismatch along a virtual fiber. Dashed line: Numerically retrieved map.

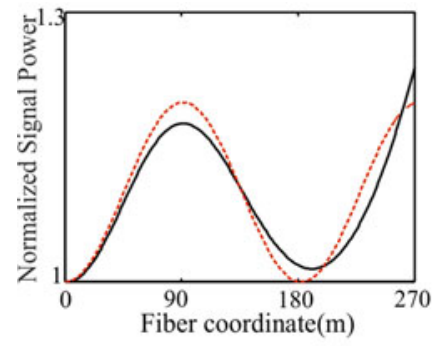

(a)

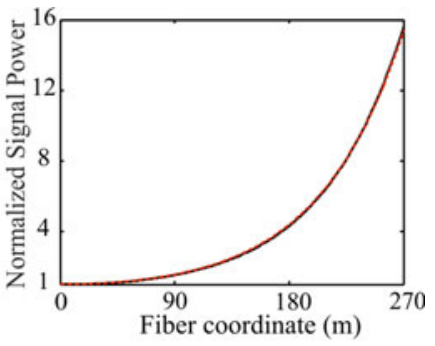

(b)

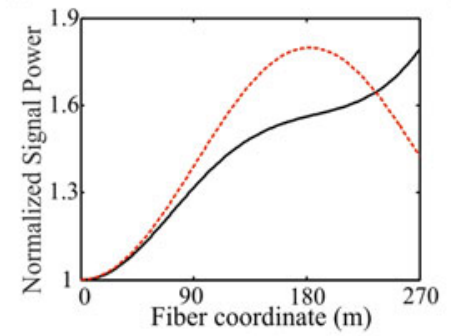

(c)

Fig. 3. Solid line: Normalized signal power $\eta(\mathrm{z})$ for the dispersion map of Fig. 2 when: (a) $\Delta \lambda>\Delta \lambda_{0}$, (b) $\Delta \lambda<\Delta \lambda_{0}$, and (c) $\Delta \lambda=\Delta \lambda_{0}$. Dotted line: The best fit of the normalize signal power when the dispersion coefficient is constant.

the wavelength of the degenerate pump of the FWM Eqs. (1) and (2) is set to $\lambda_{p}=1558 \mathrm{~nm}$. The pump power is $700 \mathrm{~mW}$ and three different wavelength positions are considered for the signal.

First, the wavelength detuning of the pump and the signal is set to $\Delta \lambda=26.3 \mathrm{~nm}$. The distribution of the signal power normalized to the initial signal power, i.e., $\eta(z)=P_{s}(z) / P_{s}(0)$ is calculated by numerically solving Eqs. (1) and (2) using a commercial software package [31]. The calculated normalized signal power is shown in Fig. 3(a) (solid line). Since the calculated $\eta(z)$ in Fig. 3(a) is almost sinusoidal, the signal power distribution is close to the signal power distribution along an ideal fiber with no dispersion fluctuation [16], [17]. In other words, the presence of dispersion fluctuation is not strongly reflected in the signal power distribution. This point 
can be easily demonstrated by calculating the distribution of the normalized signal power, $\eta(z)$, in a fiber with a constant dispersion $D(\lambda=1558 \mathrm{~nm})=0.09 \mathrm{ps} /(\mathrm{nm} \cdot \mathrm{km})$. The difference between $\eta(z)$ of a fiber with $D(\lambda=1558 \mathrm{~nm})$ and $\eta(z)$ of the original fiber is less than $2 \%$. Therefore, zero and nonzero vertical resolutions in the dispersion have mapped to almost the same level of vertical resolution in power. In other words, this spectral positioning clearly diminishes the vertical resolution of dispersion and has low sensitivity. Practically, it is almost impossible to extract $\delta D(z)$ from $\eta(z)$ insofar as $\eta(z)$ can be generated by constant dispersion.

Second, the wavelength detuning of the pump and signal is set to $\Delta \lambda=16 \mathrm{~nm}$. This time, the distribution of the signal power is almost hyperbolic. Once again, the presence of dispersion fluctuations is a matter of no consequence, and similar signal power distribution can be achieved in a fiber with constant dispersion. Normalized signal power distribution, $\eta(\mathrm{z})$, is plotted for the original fiber with dispersion fluctuation, and a fiber with constant dispersion $D(\lambda=1558 \mathrm{~nm})=0.09 \mathrm{ps} /(\mathrm{nm} \cdot \mathrm{km})$. The difference between the obtained results plotted in Fig. 3(b) is no more than $1 \%$ and again this spectral positioning does not show enough sensitivity.

It is worth noting that the two abovementioned values for the wavelength detuning between the pump and signal correspond to two different regimes: the sinusoidal FWM power exchange for $\Delta \lambda=26.3 \mathrm{~nm}$, and phase insensitive parametric amplification for $\Delta \lambda=16 \mathrm{~nm}$. Neither of these two regimes is sensitive to the presence of dispersion fluctuations. There is, however, a value for the wavelength detuning at which the presence of dispersion fluctuations becomes a matter of great consequence. This critical value for the wavelength detuning of the pump and signal is $\Delta \lambda_{0}=23.4 \mathrm{~nm}$ for our particular example. This time the minimum attainable difference between $\eta(\mathrm{z})$ of the fibers with and without dispersion fluctuations is as large as $12 \%$. Interestingly, the normalized signal power distribution plotted in Fig. 3(c), resembles neither the oscillatory (sinusoidal) FWM power exchange scheme observed at $\Delta \lambda=26.3 \mathrm{~nm}$, nor the non-oscillatory (hyperbolic) parametric amplification observed at $\Delta \lambda=16 \mathrm{~nm}$.

The critical value for the wavelength detuning of pump and signal at which the normalized signal power distribution becomes sensitive to dispersion fluctuations is as follows:

$$
\Delta \lambda_{o}=2 \lambda_{o}\left(\frac{\gamma P}{2 \pi c D_{o}\left(\lambda_{o}\right)}\right)^{1 / 2}
$$

Thisdetuning value corresponds to $|\Delta \beta|=4 \gamma P$ in Eq. (3). At this detuning value, the signal wavelength is at the edge of the parametric simplification bandwidth of a fiber without dispersion variations or $\delta D(z)=0$. The sensitivity of the normalized signal power distribution to dispersion fluctuations at this particular detuning value should not come as a surprise since dispersion fluctuations can drastically change the pattern of the normalized signal power distribution when the signal lies at the edge of the parametric simplification bandwidth. The normalized signal power distribution can be changed from the parabolic power distribution [16] to either the oscillatory pattern of the FWM power exchange, or the non-oscillatory pattern of parametric amplification. In other words, the presence of the dispersion fluctuations deviates the dispersion from its average value $D_{0}(\lambda)$, and thus moves the sideband wavelengths and causes transition from the oscillatory FWM power exchange regime to the non-oscillatory parametric amplification regime. That is why the normalized signal power distribution becomes sensitive to dispersion fluctuations. It is worth noting that observing anomalous signal power distribution along the fiber that does not match the signal power distribution predicted by FWM differential equations in a fiber with uniform dispersion coefficient has been experimentally reported in [26] for the signal wavelengths tuned to the edge of the MI spectrum and this numerical example confirms how tiny fluctuations of dispersion result in discernable variations in the distribution of the FWM generated power for the mentioned spectral positioning.

To assess the accuracy of deriving the dispersion map using distributed FWM generated power, when signal lies at the edge of parametric amplification bandwidth, a simulate-then-retrieve method [15] is implemented. In the retrieve procedure it is attempted to minimize the following cost function by searching for the proper map $\Delta \beta_{r}(z)$.

$$
F=\left\|\eta^{\Delta \beta_{r}(z)}(z)-\eta^{\Delta \beta(z)}(z)\right\|
$$

where $\eta^{\Delta \beta(z)}(z)$ and $\eta^{\Delta \beta r(z)}(z)$ are the normalized signal power distribution satisfying Eqs. (1) and (2) for the generated $\Delta \beta(z)$ and retrieved $\Delta \beta_{r}(z)$, respectively. Also, $\|$.$\| denotes the$ Euclidian norm. The details of this procedure is explained in the appendix. The dispersion map which eventually yields the minimum $F$ is shown in Fig. 2. The reconstructed map is in great agreement with the actual map particularly after first few meters for which the idler has been built up. As the result of enhanced sensitivity, the normalized first order norm of the difference between the actual and the retrieved dispersion maps is turned out to be $0.1 \%$ while it has been reported as almost $5 \%$ for the method described in [15].

\section{EXPERIMENTAL RESULTS}

The experiment is carried out for a 500 m-long HNLF with reported average value of ZDW of $1551.4 \mathrm{~nm}$ and $\gamma$ of $10 \mathrm{~W}^{-1} \mathrm{~km}^{-1}$ placed as the FUT in the experimental setup of Fig. 1. The pump power and wavelength are $680 \mathrm{~mW}$ and $1553.6 \mathrm{~nm}$, respectively. The pulse peak power of the signal is held around $20 \mathrm{~mW}$ which ensures non-depleted regime [16], [17]. In compliance with the proposed scheme, the detuning between the wavelengths of the signal and the pump is set to match the critical wavelength detuning $\Delta \lambda_{0}$. This step is carried out by inspecting the MI spectrum as observed in the OSA. Therefore, the signal wavelength is at $1574.6 \mathrm{~nm}$, which coincides with the edge of the MI spectrum as shown in Fig. 4(a), where the output spectrum including the wavelengths of pump, signal, and idler together with the MI spectrum are plotted. To demonstrate that the edge of the MI spectrum matches the edge of the parametric gain spectrum, the average value of the dispersion of the FUT is first extracted from the MI spectrum [16] and is found to be $D_{0}(1553.6 \mathrm{~nm})=0.08 \mathrm{ps} /(\mathrm{nm} \cdot \mathrm{km})$. 


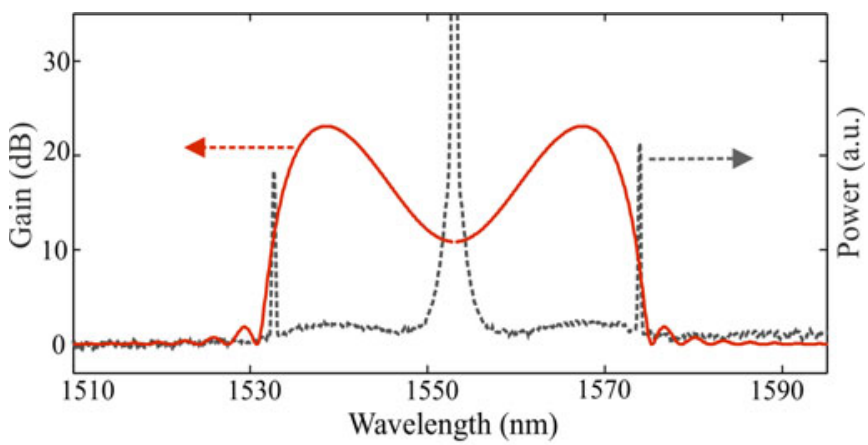

(a)

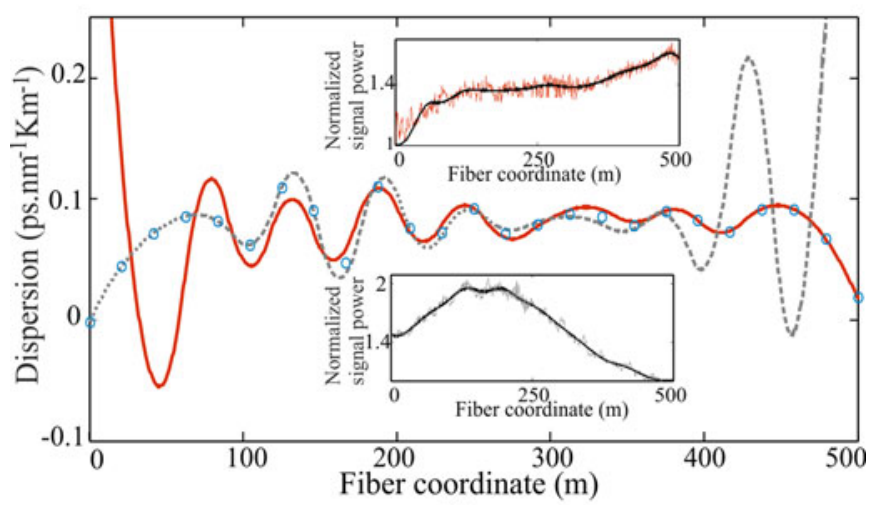

(b)

Fig. 4. (a) MI spectrum of the fiber (dotted grey), and the corresponding gain spectrum when the dispersion of the fiber is constant (red). (b) Extracted dispersion maps when the signal is launched from the left (red line) and the right (dashed grey), and the proposed dispersion map (bullets). Insets: Measured normalized signal power when the signal is launched from the left (dotted red) and the right (dotted grey), and reconstructed normalized signal powers when the signal is launched from the left and the right are plotted in grey lines in their relevant insets.

The gain spectrum of a fiber with uniform dispersion coefficient $D_{0}(1553.6 \mathrm{~nm})=0.08 \mathrm{ps} /(\mathrm{nm} \cdot \mathrm{km})$ is then plotted in Fig. 4(a), where it can be compared against the MI spectrum. It can be easily seen that the signal and pump wavelengths are correctly detuned.

The CW probes are fed through the other end of the fiber, and the distribution of the normalized signal power $\eta(z)$, is experimentally derived by using Eq. (8). Finally, an optimization algorithm for minimizing $F$ in Eq. (10) is employed and the dispersion map is extracted [31]. The details of the dispersion reconstructed algorithm is given in appendix.

As already expected, the obtained results are erroneous in the vicinity of the launching point of the pump and signal. This issue is overcome by repeating the experiment when the pump and signal are launched into the other end of the FUT $(z=$ 500 meter). The obtained results are shown in Fig. 4(b). The extracted dispersion maps between $z=100 \mathrm{~m}$ and $z=400 \mathrm{~m}$ are in very good agreement with each other. The difference is less than $2 \%$. Since the experiments run at two different directions are independent from each other, the good agreement between the extracted dispersion maps stands witness for the reliability of the obtained results. One might argue that the most accurate dispersion map for $100<z<400$ is the average value of the dispersion maps obtained by running the experiments at opposite ends. For $0<z<100$, and for $400<z<500$, on the other hand, the accurate dispersion map is the one which is obtained when the pump and signal are launched from $z=500 \mathrm{~m}$, and from $z=0$, respectively.

The extracted dispersion maps $D\left(z, \lambda_{p}\right)$ are further used for the reconstruction of $\eta(z)$. The reconstructed $\eta(z)$ s can be compared against the experimentally derived $\eta(z)$ plotted in the insets of Fig. 4(b), resulting in a difference of less than $2.5 \%$.

\section{CONCLUSION}

A novel method for dispersion map extraction along HNLFs is proposed. It is based on the BOTDA measurement of the FWMgenerated power and has obtained the highest level of accuracy in comparison with other existing methods. The accuracy of the method is indebted to the fact that the sidebands of the FWM interaction are tuned to coincide with the edge of the MI spectrum.

The proposed scheme, relying on a simple two-shot experiment, is tested on a typical HNLF and accurate results are obtained. While the destructive method of chopping the fiber into subsequent segments [11], [12] to validate the dispersion map has been discarded for practical reasons, the accuracy of the extracted map has been validate here by two independent approaches. One is to use two independent experiment runs through opposite ends of the fiber $(z=0$, and $z=L)$ which have yielded almost identical results. The other approach is the numerical study of the proposed method which has demonstrated the accuracy of the reconstruction dispersion with less than $2 \%$ error for the dispersion extraction from bidirectional measurements.

\section{APPENDIX}

The normalized signal power distribution measured along the fiber is not a single-valued function of the dispersion fluctuations $\delta D(\mathrm{z})$. This point is validated by showing that the normalized signal power distribution is a multiple-valued function of the linear propagation constant mismatch $\Delta \beta(\mathrm{z})$. First, it should be noted that both $\theta(z)$ and $\pi-\theta(z)$ result in identical patterns for the normalized signal power distribution. Then, the linear propagation constant mismatch $\Delta \beta(\mathrm{z})$ and the relative phase mismatch term $\theta(z)$ in the non-depleted regime [16] are related to each other via the following equation:

$$
d \theta / d z=\Delta \beta(z)+2 \gamma P+\gamma P\left(\frac{P_{s}+P_{I}}{\sqrt{P_{s} P_{I}}}\right) \cos (\theta)
$$

Therefore, both $\left.\Delta \beta_{(} z\right)$ corresponding to $\theta(z)$, and $\Delta \beta_{2}(z)=$ $\left.-4 \gamma P_{p}-\Delta \beta_{(} z\right)$ which corresponds to $\theta(z)=\pi-\theta(z)$, generate the same patterns for the normalized signal power distribution. Now, if the search for the linear relative phase mismatch term is limited to smooth functions, there are at least two permissible functions $\theta(z)$ and $\pi-\theta(z)$ that can generate the same power distribution. These two linear relative phase mismatch terms correspond to two different continuous functions for the linear propagation constant mismatch $\Delta \beta(z)$. But if the search for the linear relative phase mismatch term is limited to continuous but non-smooth functions, there might be more 
than two linear relative phase mismatch terms that can generate the same power distribution. This is on account of the fact that whenever smooth functions $\theta(z)$ and $\pi-\theta(z)$ meet each other (they meet each other when one of them becomes either $+\pi / 2$ or $-\pi / 2$ ), two new sets of continuous but non-smooth functions are conceivable for the relative phase mismatch term. As a matter of fact, if there are $m$ points at which the linear relative phase mismatch term becomes $+\pi / 2$ and $n$ points at which it becomes $-\pi / 2$, there would be $2^{m+n+1}$ continuous but non-smooth functions for the linear relative phase mismatch term, which generate the same normalized signal power distribution. Non-smooth linear relative phase mismatch terms, however, correspond to non-smooth functions for the linear propagation constant mismatch $\Delta \beta(z)$. Since non-smooth $\Delta \beta(z)$ is not physical, all $2^{m+n+1}$ continuous but non-smooth functions for the linear relative phase mismatch term must be discarded. It should be noted that the continuous but non-smooth linear relative phase mismatch functions are usually non-differentiable because $\theta(z)$ and $\pi-\theta(z)$ have oppositely-signed derivatives when they cross each other at $+\pi / 2$ or $-\pi / 2$. Under particular circumstances however, when the derivative of the relative phase mismatch term is zero, i.e., when $\Delta \beta(z)=-2 \gamma P_{p}$, $\theta(z)$ and $\pi-\theta(z)$ just touch each other and generate differentiable but non-smooth functions.

The preceding argument shows that the brute-force numerical search for either $\Delta \beta(z)$ or $\theta(z)$ is bound to be troublesome because neither $\Delta \beta(z)$ nor $\theta(z)$ is uniquely determined by a certain distribution for the normalized signal power. To resolve this issue, the brute-force numerical search is carried out to find $\sin (\theta(z))$, which is uniquely determined by the normalized signal power. Then, the sought-after $\Delta \beta(z)$ is analytically extracted from the numerically found $\sin (\theta(z))$. In this fashion, the numerical issues due to the fact that the normalized signal power distribution is a multiple-valued function of $\Delta \beta(z)$ are avoided. This point is numerically demonstrated below.

An optical fiber of length $L=500 \mathrm{~m}$, nonlinear coefficient of $\gamma=11 \mathrm{~W}^{-1} \mathrm{~km}^{-1}$, and a specific linear propagation constant mismatch $\Delta \beta(z)$ as shown by the solid grey line in Fig. A.1(a) is considered. The optical pump power is assumed to be $P_{P}=0.5 \mathrm{~W}$. Given that the linear propagation constant mismatch $\Delta \beta(z)$ is specified, the corresponding relative phase mismatch term, $\theta_{1}(z)$, its sine, $\sin \left(\theta_{1}(z)\right)$, and the distribution of the normalized signal power along the fiber $\eta(\mathrm{z})$, are all uniquely determined and plotted by solid lines in Fig. A.1(b)-(d), respectively. It should be however noted that even though there is a one-to-one correspondence between $\eta(z)$ and $\sin (\theta(z))$ whereby the latter can be uniquely determined from the former, no one-to-one relationship is held between $\theta(z)$, and $\sin (\theta(z))$ since $\theta_{2}(z)=\pi-\theta_{1}(z)$ whose sine is no different from $\sin \left(\theta_{1}(z)\right)$ yields the same $\eta(z)$. Fig. A.1(b) shows that the relative phase mismatch term becomes $\pi / 2$ at $z=100 \mathrm{~m}$, where $\theta_{2}(z)=\pi-\theta_{1}(z)$ touches the relative phase mismatch term $\theta_{1}(z)$. Therefore, the following continuous yet non-smooth relative phase mismatch terms are also conceivable:

$$
\theta_{3}(z)= \begin{cases}\theta_{1}(z) & \text { for } 0<z<100 \\ \pi-\theta_{1}(z) & \text { for } 100<z<500\end{cases}
$$

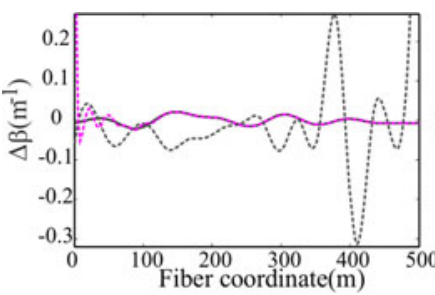

(a)

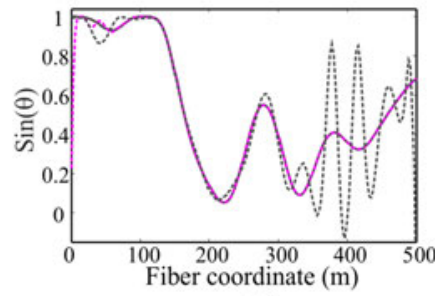

(c)

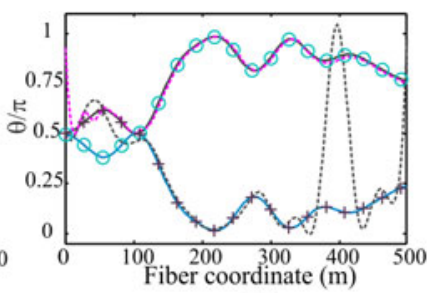

(b)

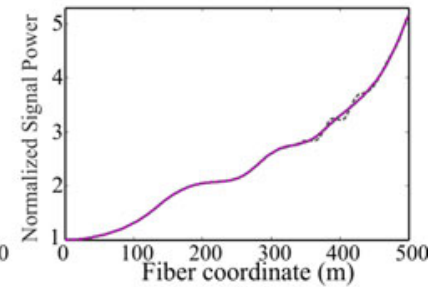

(d)
Fig. A.1. (a) Actual $\Delta \beta(z)$ (solid grey), $\Delta \beta(z)$ obtained by minimizing Eq. (A.2) (dashed grey) and obtained by minimizing Eq. (A.3) (dashed pink). (b) $\theta_{1}(z)$ (solid grey), $\theta_{2}(z)$ (solid cyan), $\theta_{3}(z)$ (crosses) and $\theta_{4}(z)$ (circles). $\theta(z)$ which is obtained by minimizing Eq. (A.2) (dashed grey) and Eq. (A.3) (dashed pink). (c) $\sin (\theta(z))$ which satisfies Eqs. (1) and (2) (solid grey), $\sin (\theta(z))$ which is obtained by minimizing Eq. (A.2) (dashed grey) and Eq. (A.3) (dashed pink). (d) Normalized signal power based on the actual $\Delta \beta(z)$ (solid grey), $\Delta \beta(z)$ which minimizes Eq. (A.3) (dashed pink) and $\sin (\theta(z))$ which minimizes Eq. (A.2) (dashed grey).

and

$$
\theta_{4}(z)= \begin{cases}\pi-\theta_{1}(z) & \text { for } 0<z<100 \\ \theta_{1}(z) & \text { for } 100<z<500\end{cases}
$$

These two non-smooth relative phase mismatch terms are plotted together with $\pi-\theta_{1}(z)$ in Fig. A.1(b).

The major difficulty arisen from this issue is that $\theta_{1}(z), \theta_{2}(z)$, $\theta_{3}(z)$ and $\theta_{4}(z)$ result in different linear propagation constant mismatch $\Delta \beta(z), \Delta \beta_{2}(z), \Delta \beta_{3}(z)$ and $\Delta \beta_{4}(z)$, even though they all have the same $\sin (\theta(z))$ and the same $\eta(z)$. It is worth noting that while $\Delta \beta(z)$ and $\Delta \beta_{2}(z)$ are smooth functions, $\Delta \beta_{3}(z)$ and $\Delta \beta_{4}(z)$ are non-smooth functions.

Now, any direct attempt toward extracting the linear propagation constant mismatch $\Delta \beta(\mathrm{z})$ by running a brute-force numerical search generally strives to minimize the following cost function:

$$
F^{\Delta \beta}=\left\|\eta(z)-\eta^{\Delta \beta(z)}(z)\right\|
$$

where $\eta^{\Delta \beta(z)}(z)$ is the normalized signal power distribution satisfying Eqs. (1) and (2) for a given $\Delta \beta(z)$. Since $\Delta \beta(z), \Delta \beta_{2}(z), \Delta \beta_{3}(z)$, and $\Delta \beta_{4}(z)$ all minimize $F^{\Delta \beta}$, it is no wonder that using the least mean square method for extraction of $\Delta \beta(z)$ by minimizing $F^{\Delta \beta}$ results in an erroneous linear propagation constant mismatch which is depicted in Fig. A.1(a). This erroneous linear propagation constant mismatch, however, provides a good estimate of the normalized power distribution. This point can be easily verified by comparing $\eta^{\Delta \beta(z)}(z)$ with $\eta(z)$ in Fig. A.1(d). Therefore, the failure of the least mean square method for extraction of $\Delta \beta(z)$ is not due to the failure in minimization of the cost function $F^{\Delta \beta}$, but is on account of the fact that the existence of multiple answers $\Delta \beta(z), \Delta \beta_{2}(z)$, 
$\Delta \beta_{3}(z)$, and $\Delta \beta_{4}(z)$ befuddles the inverse algorithm. This issue can be easily overcome by running a brute-force numerical search, which strives to minimize the following cost function:

$$
F^{\sin (\theta)}=\left\|\eta(z)-\eta^{\sin (\theta(z))}(z)\right\|
$$

where $\eta^{\sin (\theta(z))}(z)$ is the normalized signal power distribution satisfying Eq. (1). This time, the cost function is minimized by searching for the appropriate $\sin (\theta(z))$. As demonstrated in Fig. A.1(d), $\eta^{\sin (\theta(z))}(z)$ virtually reconstructs $\eta(z)$. However, the success of this approach is indebted to the fact that $\sin (\theta(z))$, in contrast to $\theta(z)$, has a unique distribution. Once the appropriate $\sin (\theta(z))$ is found, all four different candidates for $\theta(z)$ are at hand. Two of them are differentiable and provide two different $\Delta \beta(z) s$ that are continuous (see Eq. (A.1)). The correct $\Delta \beta(z)$ is the one whose corresponding $D\left(z, \lambda_{P}\right)$ has the correct sign. Thus, the extracted $\Delta \beta(z)$ plotted in Fig. A.1(a) is in great agreement with the original distribution of the linear propagation constant mismatch, even though it is erroneous along the first few meters of the fiber. This is due to the fact that there is a singularity at $z=0$ in Eq. (A.2). This mathematical singularity is caused by the absence of idler at the input of the fiber. Fortunately, this issue can be overcome by launching the signal from the other end of the fiber, i.e. $z=L$.

\section{ACKNOWLEDGMENT}

The authors would like to thank Prof. Luc Thévenaz and Dr. Mehdi Alem from the Group for Fiber Optics, Ecole Polytechnique Fédérale de Lausanne for their fruitful discussion and insightful suggestions.

\section{REFERENCES}

[1] A. Mussot et al., "Zero-dispersion wavelength mapping in short singlemode optical fibers using parametric amplification," IEEE Photon Technol. Lett., vol. 18, no. 1, pp. 22-24, Jan. 2006.

[2] K. Inoue, "Four-wave mixing in an optical fiber in the zero-dispersion wavelength region," J. Lightw. Technol., vol. 10, no. 11, pp. 1553-1561, Nov. 1992

[3] B. P. Kuo and S. Radic, "Highly nonlinear fiber with dispersive characteristic invariant to fabrication fluctuations," Opt. Express, vol. 20, no. 7, pp. 7716-7725, Mar. 2012.

[4] A. Mohajerin-Ariaei et al., "Phase noise mitigation of QPSK signal utilizing phase-locked multiplexing of signal harmonics and amplitude saturation," Opt. Lett., vol. 40, no. 14, pp. 3328-3331, Jul. 2015.

[5] M. Hirano et al., "Silica-based highly nonlinear fibers and their application," IEEE J. Sel. Topics Quantum Electron., vol. 15, no. 1, pp. 103-113, Jan. 2009.

[6] E. Myslivets et al., "A new class of high-resolution measurements of arbitrary-dispersion fibers: Localization of four-photon mixing process," J. Lightw. Technol., vol. 27, no. 3, pp. 364-375, Feb. 2009.

[7] E. Myslivets and S. Radic, "Spatially resolved measurements of the chromatic dispersion in fibers," J. Lightw. Technol., vol. 33, no. 3, pp. 597-608, Feb. 2015

[8] M. Karlsson, "Four-wave mixing in fibers with randomly varying zerodispersion wavelength," J. Opt. Soc. Amer. B, vol. 15, no. 8, pp. 2269-2275, Aug. 1998.

[9] F. Yaman et al., "Impact of dispersion fluctuations on dual-pump fiberoptic parametric amplifiers," Photon. Technol. Lett., vol. 16, no. 5, pp. 1292-1294, May 2004.

[10] P. Velanas, A. Bogris, and D. Syvridis, "Impact of dispersion fluctuations on the noise properties of fiber optic parametric amplifiers," J. Lightw. Technol., vol. 24, no. 5, pp. 2171-2178, May 2006.
[11] E. Myslivets et al., "Spatial equalization of zero-dispersion wavelength profiles in nonlinear fibers," IEEE Photon. Technol. Lett., vol. 21, no. 24, pp. 1807-1809, Dec. 2009 .

[12] S. Moro et al., "Synthesis of equalized broadband parametric gain by localized dispersion mapping," IEEE Photon. Technol. Lett., vol. 20, no. 23 , pp. 1971-1973, Dec. 2008.

[13] E. Myslivets and S. Radic, "Advanced fiber optic parametric synthesis and characterization," presented at the Optical Fiber Commun./National Fiber Optic Engineers Conf., Los Angeles, CA, USA, Mar. 6, 2011, Paper OWL5.

[14] E. Myslivets, N. Alic, and S. Radic, "Spatially resolved measurement in waveguides with arbitrary chromatic dispersion," IEEE Photon. Technol. Lett., vol. 20, no. 21, pp. 1793-1795, Nov. 2008.

[15] E. Myslivets, N. Alic, and S. Radic, "High resolution measurement of arbitrary-dispersion fibers: Dispersion map reconstruction techniques," $J$. Lightw. Technol., vol. 28, no. 23, pp. 3478-3487, Dec. 2010.

[16] M. E. Marhic, Fiber Optical Parametric Amplifiers, Oscillators and Related Devices. New York, NY, USA: Cambridge Univ. Press, 2008.

[17] G. P. Agrawal, Nonlinear Fiber Optics. San Diego, CA, USA: Academic, 2007

[18] E. Myslivets, N. Alic, and S. Radic, "Strict localization of nonlinear interactions in optical fibers by subsequent Brillouin amplification and attenuation," IEEE Photon. Technol. Lett., vol. 3, no. 22, pp. 170-172, Feb. 2010.

[19] M. Eiselt, R. M. Jopson, and R. H. Stolen, "Nondestructive positionresolved measurement of the zero-dispersion wavelength in an optical fiber,” J. Lightw. Technol., vol. 15, no. 1, pp. 135-143, Jan. 1997.

[20] J. Gripp and L. F. Mollenauer, "Enhanced range for OTDR-like dispersion map measurements," Opt. Lett., vol. 23, no. 20, pp. 1603-1605, Oct. 1998.

[21] K. Y. Song, M. González Herráez, and L. Thévenaz, "Mapping of chromatic-dispersion distribution along optical fibers with 20-m spatial resolution," J. Lightw. Technol., vol. 23, no. 12, pp. 4140-4146, Dec. 2005.

[22] M. González-Herráez et al., "Retrieval of the zero-dispersion wavelength map of an optical fiber from measurement of its continuous-wave fourwave mixing efficiency," Opt. Lett., vol. 27, no. 17, pp. 1546-1548, Sep. 2002.

[23] T. Horiguchi and M. Tateda, "BOTDA-non-destructive measurement of single-mode optical fiber attenuation characteristics using Brillouin interaction: Theory," J. Lightw. Technol., vol. 7, no. 8, pp. 1170-1176.

[24] Y. Koyamada et al., "Fiber-optic distributed strain and temperature sensing with very high measurand resolution over long range using coherent OTDR," J. Lightw. Technol., vol. 27, no. 9, pp. 1142-1146, May 2009.

[25] A. Vedadi et al., "Brillouin optical time-domain analysis of fiber-optic parametric amplifiers," IEEE Photon. Technol. Lett., vol. 19, no. 3, pp. 179-81, Feb. 2007.

[26] F. Alishahi et al., "Power evolution along phase-sensitive parametric amplifiers: An experimental survey," Opt. Lett., vol. 39, no. 21, pp. 6114 6117, Oct. 2014.

[27] M. Alem, M. A. Soto, and L. Thévenaz, "Analytical model and experimental verification of the critical power for modulation instability in optical fibers," Opt. Express, vol. 23, no. 23, pp. 29514-29532, Nov. 2015.

[28] J. C. Beugnot, M. Tur, S. F. Mafang, and L. Thévenaz, "Distributed Brillouin sensing with sub-meter spatial resolution: Modeling and processing," Opt. Express, vol. 19, no. 8, pp. 7381-7397, Apr. 2011.

[29] A. Zadok, E. Zilka, A. Eyal, L. Thevenaz, and M. Tur, "Vector analysis of stimulated Brillouin scattering amplification in standard single-mode fibers," Opt. Express, vol. 16, no. 26, pp. 21692-21707, Dec. 2008.

[30] B. Stiller et al., "Demonstration of polarization pulling using a fiber-optic parametric amplifier," Opt. Express, vol. 20, no. 24, pp. 27248-27253, Nov. 2012.

[31] MathWorks, Inc., MATLAB and Statistics Toolbox Release 2012b. Natick, MA, United States: MathWorks, Inc.

Authors' biographies not available at the time of publication. 